Abstracta Iranica Abstracta Iranica

Revue bibliographique pour le domaine irano-aryen

Volume 42-43 | 2021

Comptes rendus des publications de 2019-2020

\title{
Bruno Genito, Giulio Maresca (eds.). Ceramics and the Archaeological Achaemenid Horizon. Near East, Iran and Central Asia
}

\section{Sébastien Gondet}

\section{(2) OpenEdition Journals}

Édition électronique

URL : https://journals.openedition.org/abstractairanica/53587

DOI : 10.4000/abstractairanica.53587

ISSN : 1961-960X

Éditeur :

CNRS (UMR 7528 Mondes iraniens et indiens), Éditions de l'IFRI

Référence électronique

Sébastien Gondet, «Bruno Genito, Giulio Maresca (eds.). Ceramics and the Archaeological Achaemenid Horizon. Near East, Iran and Central Asia ", Abstracta Iranica [En ligne], Volume 42-43 | 2021, document 74, mis en ligne le 30 décembre 2021, consulté le 27 décembre 2022. URL : http:// journals.openedition.org/abstractairanica/53587 ; DOI : https://doi.org/10.4000/abstractairanica. 53587

Ce document a été généré automatiquement le 27 décembre 2022.

Tous droits réservés 


\title{
Bruno Genito, Giulio Maresca (eds.). Ceramics and the Archaeological Achaemenid Horizon. Near East, Iran and Central Asia
}

\author{
Sébastien Gondet
}

\section{RÉFÉRENCE}

Bruno Genito, Giulio Maresca (eds.). Ceramics and the Archaeological Achaemenid Horizon. Near East, Iran and Central Asia. Napoli : Università degli Studi di Napoli “L'Orientale", 2019, XIX-285p. (Series Minor LXXXVI \& ISMEO Serie Orientale Roma 14 / Orientalia Romana 1).

Cet ouvrage collectif rassemble les actes d'une table ronde regroupant des archéologues italiens qui s'est déroulée le 25 janvier 2016 à l'Université « L'Orientale » de Naples dont le titre original en anglais était: The Achaemenid Horizon in the Light of Ceramic Data: Production-related Issues and Cultural Interactions from the Ancient Near East to Central Asia. Les communications portaient donc sur le thème de la caractérisation des assemblages de céramiques datés de l'époque de la domination achéménide dans plusieurs provinces à l'intérieur ou en périphérie de l'empire. L'objectif général était d'abord de déterminer l'existence, ou l'absence, de variations typologiques de la céramique entre les $\mathrm{VI}^{\mathrm{e}} \mathrm{et}_{\mathrm{IV}}^{\mathrm{e}}$ s. av. n.è. Il s'agissait ensuite d'étudier la possibilité de les associer aux changements géopolitiques globaux ou plutôt à des dynamiques locales et indépendantes de l'avènement de l'empire. Le volume rassemble 11 articles répartis de la manière suivante : une introduction générale à la thématique du volume, neuf études de cas régionales, une conclusion rassemblant remarques et propositions inspirées par le contenu des différents chapitres. L'ouvrage se termine sur une bibliographie générale rassemblant les références citées dans les articles. 
2 L'importance de cette publication dans le cadre des études achéménides est exposée dans la conclusion rédigée par Rémy Boucharlat qui constitue en quelque sorte un compte rendu détaillé de cet ouvrage. Ainsi qu'il le souligne, ce volume constitue, à notre connaissance, le premier et le seul essai de typo-chronologie céramique comparée mené de manière si précise à l'échelle de pratiquement tout l'empire. Il devra donc désormais compter parmi les ouvrages de référence à consulter et à citer dans le cadre d'études sur la céramique achéménide, ou plutôt sur la céramique d'époque perse pour utiliser l'appellation que suggère Boucharlat. En effet, ce dernier plaide pour attribuer une dénomination homogène à ces assemblages céramiques très variés au sein desquels les composantes et influences achéménides, c'est-à-dire venant du centre de l'empire et de la sphère royale et/ou aristocratique, sont souvent très difficiles à détecter. Cette appellation très large et consensuelle ne viendrait nullement remplacer les terminologies et typologies locales préexistantes (Yaz III, Age du Fer III ou IV, postassyrien etc...), qui toutes sont pertinentes dans leur contexte géographique particulier. Son utilisation permettrait toutefois à la communauté archéologique de dialoguer en utilisant des termes communs et, beaucoup plus pratiquement, de retrouver plus aisément des références au cours de recherches bibliographiques. Cette nécessaire clarification et homogénéisation terminologique est également un des thèmes abordés dans l'introduction de Bruno Genito qui par ailleurs rappelle quelques grandes généralités sur les apports et les limites de l'étude du matériel céramique en archéologie.

3 La répartition géographique des études rassemblées dans ce volume est avant tout dictée par la présence d'équipes et de projets italiens, pour certains toujours actifs, auxquels les auteurs participent ou sont étroitement liés. Les régions couvertes dessinent un arc ouest-est et témoignent de la forte dynamique de recherche italienne sur l'archéologie des "mondes iraniens» (persianate) au sens très large: Anatolie occidentale (article d'Alessandro Poggio), Nord de l'Iraq/Assyrie (article de Rocco Palermo), Nord-ouest de l'Iran et pays du Petit Caucase (articles de Roberto Dan et al. et de Manuel Castelluccia), Sud du Turkménistan/vallée de l'Atrek (article de Jacopo Bruno), Tadjikistan et Est de l'Ouzbekistan/Sogdiane (article de Fabiana Raiano), Sud du Kazakhstan/delta du Syr-Daria (article de Gian Luca Bonora), Ouest du Pakistan/ Gandhara (article de Elisa Iori).

4 Tous les articles intègrent des synthèses très utiles concernant l'état actuel des connaissances sur les assemblages céramiques de la période perse replacés dans leur contexte régional et dans un intervalle chronologique large couvrant l'ensemble du premier millénaire. Etant donné la diversité des régions couvertes et donc des langues dans lesquelles sont publiés certaines données prises en compte, ces synthèses mettent donc à la disposition du plus grand nombre des résultats dont la diffusion a parfois été assez confidentielle.

5 Il est ensuite possible de subdiviser les articles en fonction de l'origine des données. Un premier groupe est constitué de synthèses rassemblant une riche documentation archéologique provenant de multiples sites ou régions. Elles se concentrent sur l'analyse des évolutions morphologiques et de la répartition de types de céramiques parfois considérés comme caractéristiques de la période achéménide. Il s'agit des articles de : A. Poggio sur les occurrences de bols carénés en Anatolie occidentale ; R. Dan et al sur l'apparition et la diffusion des jarres à anses à cornes dans le Caucase et au 
nord-ouest de l'Iran; M. Castelluccia sur la question de la diffusion de la triangle ware dans ces mêmes régions.

Le second groupe d'articles poursuit les mêmes objectifs mais en mobilisant des données de première main issues de projets archéologiques italiens pour certains encore en activité ou, pour d'autres, mis en sommeil mais dont les résultats restent en cours d'analyse. Il s'agit des articles de: R. Palermo sur les données issues des prospections en cours autour de Ninive et la difficile caractérisation de la période achéménide; J. Bruno sur les données issues de prospections des années 1970 dans la vallée de l'Atrek et l'association dans cette région de plusieurs traditions céramiques; G. Maresca sur les archives des fouilles de Dahan-e Gholaman et leur utilisation pour lancer de nouvelles études sur la période perse sur l'est de l'Iran; F. Raiano sur les données des prospections dans la vallée de Samarkand et la quasi-absence identifiée d'un horizon céramique achéménide; G. L. Bonora sur les résultats de ses recherches dans le delta du Syr-Daria et les quelques indices d'influences perses dans le matériel funéraire ; E. Iori sur les données des fouilles dans la vallée de la Swat et l'apparition de bols carénés à partir du milieu du $1^{\text {er }}$ millénaire.

7 Une première conclusion que l'on peut tirer de ce volume est assez pessimiste. Les A. constatent que les assemblages de la période perse sont partout très difficiles à définir et ne se distinguent souvent qu'assez peu des traditions préexistantes. Alors que la céramique reste le principal matériel utilisé pour étudier les dynamiques d'occupation anciennes, on ne peut dès lors que questionner, comme d'autres l'ont déjà maintes fois fait auparavant, la capacité de l'archéologie à contribuer à l'étude de l'histoire de l'empire achéménide. Pour contrebalancer cette vision pessimiste, il est nécessaire de préciser que tous les A. s'arrêtent trop souvent, et on peut le regretter, sur leur incapacité à détecter des influences achéménides sur le matériel céramique. Cette préoccupation revient peu ou prou à traiter du sujet des relations entre le centre et sa périphérie, une thématique dont on peut remettre en question la pertinence dans le contexte d'un empire à l'organisation très multipolaire. En outre, les conclusions formulées auraient plus de poids si les assemblages céramiques du "centre ", c'est-àdire de la province de Perse, étaient mieux connus, ce qui n'est absolument pas le cas à l'heure actuelle. On regrettera ainsi l'absence dans ce volume d'un article traitant spécifiquement du Sud de l'Iran, la conclusion du volume nous apprenant qu'une communication avait été donnée sur ce thème mais n'a pas donné lieu à publication.

8 Nous devons toutefois tirer de ces articles des conclusions beaucoup plus optimistes. D'une part en croisant des données archéologiques diverses, dont celles sur la céramique, plusieurs des A. arrivent à dessiner parfois plus qu'une ébauche de l'organisation territoriale des régions étudiées à l'époque achéménide. D'autre part, pratiquement tous dressent des perspectives de recherche tout à fait pertinentes dessinant les contours de projets futurs et prometteurs qui, il faut le souhaiter, pourront se réaliser à plus ou moins brève échéance.

9 Table des matières disponible à l'adresse suivante: https://www.ismeo.eu/ceramics-and-thearchaeological-achaemenid-horizon-near-east-iran-and-central-asia/ 


\section{AUTEURS}

\section{SÉBASTIEN GONDET}

UMR 5133 CNRS-Université de Lyon 